\title{
Global response of Magnetic field and lonosonde observations to intense solar flares on 6 and 10 September 2017
}

\author{
Akiko Fujimoto ${ }^{1, *}$, Akimasa Yoshikawa ${ }^{2}$, and Akihiro Ikeda $^{3}$ \\ ${ }^{1}$ Kyushu Institute of Technology, Iizuka, Japan \\ ${ }^{2}$ International Center for Space Weather Science and Education, Kyushu University, Fukuoka, Japan \\ ${ }^{3}$ National Institute of Technology Kagoshima College, Kirishima, Japan
}

\begin{abstract}
Intense X-ray fluxes during solar flares are known to cause enhanced ionization in the Earth's ionospheric D, E and F region. This sudden change of ionospheric electron density profile is serious problem to radio wave communication and navigation system. The ground magnetograms often record the sudden change in the sunlit hemisphere during the enhanced X-ray flux, due to the sudden increase in the global ionospheric current system caused by the flare-induced enhanced ionospheric conductivity. These geomagnetic field disturbances are known as "solar flare effects" (SFEs) or geomagnetic crochets [Campbell, 2003]. The typical SFE is increase variation on the equatorial magnetic data. On Ionosonde observation during solar flare event, the High-Frequency (HF) radio wave blackout is often detected in ionogram due to the sudden disturbance in ionosphere. Two intense X-class solar flares occurred on 6 and 10 September 2017. We investigated the magnetic field and Ionosonde responses to the intense solar flare events. Dayside magnetic field variations sudden increased due to the ionospheric disturbance resulting from solar flare. There is no response in night side magnetometer data. The magnitude of SFE (magnetic field) is independent of solar flare x-ray magnitude. We found HF radio wave blackout in ionogram at dayside Ionosonde stations. The duration of blackout is dependent of latitude and local time of Ionosonde stations. There is the different feature of ionogram at night side.
\end{abstract}

\section{Introduction}

The Ionospheric electron density is affected by the solar activity, especially intense solar flares could result in the strong ionospheric disturbances. Intense X-ray fluxes during solar flares are known to cause enhanced ionization in the Earth's ionospheric D, E and F region. This sudden change of ionospheric electron density profile is serious problem to radio wave communication and navigation system. The sudden ionospheric disturbances due to solar flares result in the sudden increase in the total electron content (TEC) [e.g. 1-3], HighFrequency (HF) radio wave blackout [e.g. 4-5] and the enhancement of magnetic field variation [6-7].

* Corresponding author: fujimoto@ces.kyutech.ac.jp 
The ionization of the atmosphere and creation of planetary ionosphere are caused by the solar extreme ultraviolet (EUV) and X-ray photons. The peak enhancement in TEC has a higher correlation with peak enhancement in EUV flux than X-ray flux [8-10]. They examined the relationship between the enhancement in EUV flux $(\triangle T E C)$ and the solar zenith angle. There is a significant dependence $\triangle T E C$ on the solar zenith angle. The solar zenith angle decrease with increasing $\triangle T E C$. This suggests that the $\triangle T E C$ variation is mainly controlled by ionizing and heating atmospheric gas due to EUV flux.

The ground magnetograms often record the sudden change in the sunlit hemisphere during the enhanced X-ray flux, due to the sudden increase in the global ionospheric current system caused by the flare-induced enhanced ionospheric conductivity. These geomagnetic field disturbances are known as "solar flare effects" (SFEs) or geomagnetic crochets [11]. The typical SFE is increase variation on the equatorial magnetic data. Meza et al. (2009) reported the global response of SFE during the intense solar flare (X-class flare). They demonstrated the absolute value of total magnetic field due to the solar flare and revealed that the SFE was larger in the local morning than in the afternoon. The equatorial geomagnetic response to X-class flare is sometimes negative SFEs during morning and evening counter-electrojet (CEJ) periods [12-16]. There are few reports on negative SFEs during local noon [17-18]. Little is known about the equatorial negative SFE generation mechanism during the intense solar flare and also the relationship between EUV flux and global SFE variation. The demonstration of global SFE distribution during X-class flare is the key to solving the main source and generation mechanism of SFE.

This paper is intended as an investigation of the response of the geomagnetic solar flare effect (gsfe) to the time series of the EUV flux variation during two intense solar flares on 6 and 10 September 2017. Sec. 2 describes the observation used in this work, and Sec. 3 shows the analysis and results of gsfe. In Sec.4, we discuss the relationship between ionospheric solar flare effect (isfe) and gsfe, and summarize this work.

\section{Observation}

Two intense X-class solar flares occurred on September 6 (X9.3) and 10 (X8.2), 2017. Figure 1 shows the 1-minute averaged X-ray flux data recorded by the Geostationary Operational Environmental Satellites (GOES-13, data is available at the NOAA's GOES Space Environment Monitor, https://www.ngdc.noaa.gov/stp/satellite/goes/dataaccess.html) in the wavelength band of 0.1 to $0.8 \mathrm{~nm}$, and the 15 -seconds averaged Extreme ultraviolet (EUV) flux observed by the Solar EUV Monitor (SEM) on board the SOlar and Heliospheric Observatory (SOHO) (data is available at the the University of Southern California (USC) Space Sciences Center (SSC) web site, https://dornsifecms.usc.edu/space-sciencescenter/download-sem-data/) in the wavelength band of 26 to $34 \mathrm{~nm}$.

On September 6, X-ray flux intensity started increasing at 11:53 UT, and reached the peak value at 12:02 UT, finally the end time of X-ray solar flare is 12:20 UT. The onset time of EUV flux increase is later than the time of X-ray, at 11:54:20 UT. EUV flux rapidly increased and gradually reached the peak value. The first peak time of EUV flux previously appeared before the time of X-ray peak time $(-205$ seconds $=3 \mathrm{~m} \mathrm{25s})$. EUV flux reached the second peak after a delay of 128 seconds $(2 \mathrm{~m} 8 \mathrm{~s})$ from the time of X-ray flux peak. For 10 September, the X-ray flux intensity started increasing at 15:35 UT, and reached the peak value at 16:36 UT, finally the end time of X-ray solar flare is 16:41 UT. The onset time of EUV flux increase is about 9 minutes later than the time of X-ray, at 15:54:09 UT. The EUV flux shows a step-like increase. The first peak time of EUV is later of X-ray peak time by 150 seconds ( $2 \mathrm{~m} 30 \mathrm{~s}$ ). The EUV flux reached the second peak after a delay of 8446 seconds ( $2 \mathrm{~h} 20 \mathrm{~m} \mathrm{46s)} \mathrm{from} \mathrm{the} \mathrm{time} \mathrm{of} \mathrm{X-ray} \mathrm{flux} \mathrm{peak.} \mathrm{The} \mathrm{rise} \mathrm{of} \mathrm{the} \mathrm{X-ray} \mathrm{flux} \mathrm{continued} \mathrm{for} 9$ 
minutes and 31 minutes for September 6 and September 10, respectively. For EUV flux, the first rise and the second rise continued for about 4 minutes and 6 minutes for September 6 , about 14 minutes and 2 hours 18 minutes for September 10, respectively. The increase on the EUV flux lasted longer than the X-ray flux. Table 1 summarizes the flare time and the time when EUV flux stared increasing and reached peaks.
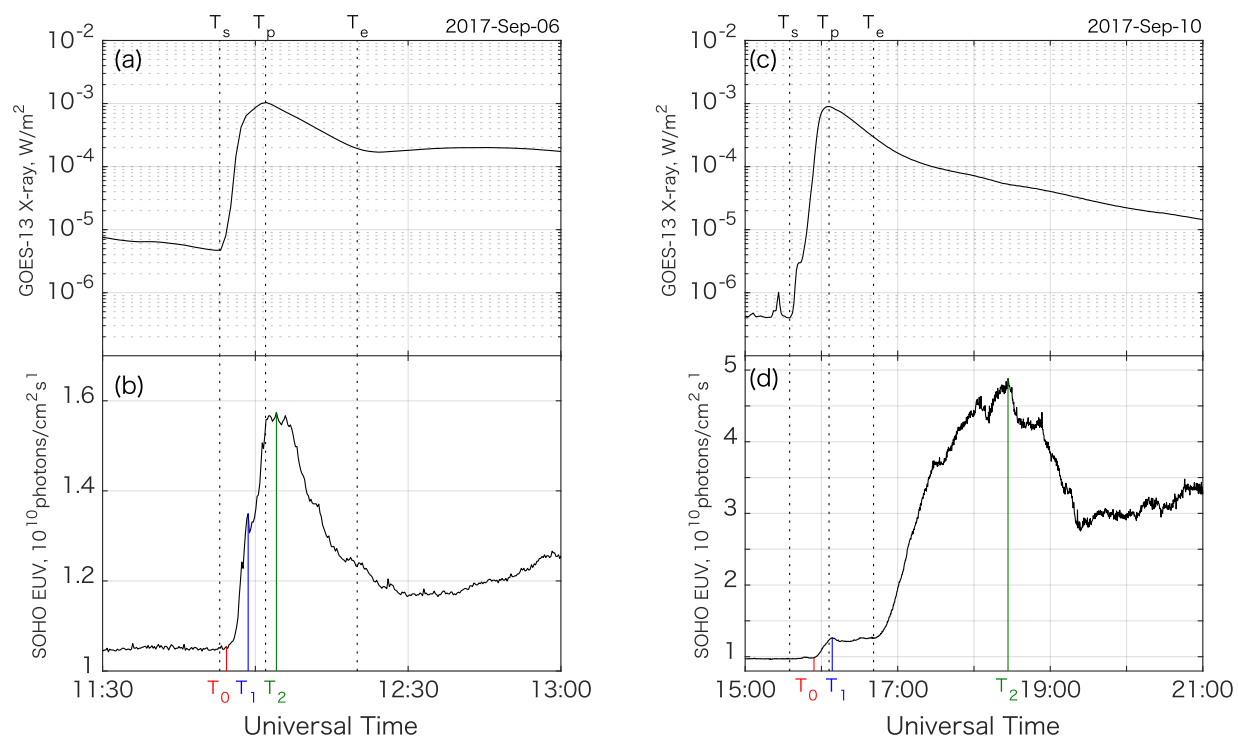

Fig. 1. Time series data of (a, c) GOES-13 X-ray flux in the 0.1- $0.8 \mathrm{~nm}$ bands and (b, d) SOHO SEM EUV flux in the 26-34 $\mathrm{nm}$. The vertical black dotted lines indicate the start time $\left(\mathrm{T}_{\mathrm{S}}\right)$, the peak time $\left(\mathrm{T}_{\mathrm{p}}\right)$ and the ned time $\left(\mathrm{T}_{\mathrm{e}}\right)$ for the $\mathrm{X}$-ray flux of the solar flares. The color solid lines indicate the start time $\left(\mathrm{T}_{0}\right)$, the first peak time $\left(\mathrm{T}_{1}\right)$ and the second peak time $\left(\mathrm{T}_{2}\right)$ for SOHO EM EUV flux.

Table 1. Solar flare events.

\begin{tabular}{|c|c|c|c|c|c|c|}
\hline \multirow{2}{*}{ Date } & \multicolumn{2}{|c|}{ GOES X-ray (h:m UT) } & \multicolumn{3}{c|}{ SOHO EUV (h:m:s UT) } \\
\cline { 2 - 7 } & $\begin{array}{c}\text { Start } \\
\text { time }\end{array}$ & $\begin{array}{c}\text { Peak } \\
\text { time }\end{array}$ & $\begin{array}{c}\text { End } \\
\text { time }\end{array}$ & $\begin{array}{c}\text { Start } \\
\text { time }\end{array}$ & $\begin{array}{c}\text { First Peak } \\
\text { time }\end{array}$ & $\begin{array}{c}\text { Second Peak } \\
\text { time }\end{array}$ \\
\hline $2017 / 09 / 06$ & $11: 53$ & $12: 02$ & $12: 20$ & $11: 54: 20$ & $11: 58: 35$ & $12: 04: 08$ \\
\hline $2017 / 09 / 10$ & $15: 35$ & $16: 06$ & $16: 41$ & $15: 54: 09$ & $16: 08: 30$ & $18: 26: 46$ \\
\hline
\end{tabular}

In this work, we used the multipoint ground-based geomagnetic field observation to demonstrate the global magnetic field response to the intense solar flare. The 1-minute vector magnetometer time series data are obtained from the SuperMAG website [19] (http://supermag.jhuapl.edu/mag/). The SuperMAG database consists of the stations from various magnetometer observation groups. Figure 2 shows the example of the magnetic filed time series data observed at Huancayo (Peru, geographic longitude: 284.67 degree, geographic latitude: -12.05 degree, magnetic longitude: -2.75 degree, magnetic latitude: 1.17 degree, MAGDAS network [20-23]) on September 6 and September 10. The local time (LT) of Huancayo are 06:52 LT for 11:53 UT on September 6 and 10:34 LT for 15:35 UT on September 10. Figure $2 \mathrm{a}$ and $2 \mathrm{c}$ show the Total B values are calculated as 


$$
\text { Total } B=\left(X^{2}+Y^{2}+Z^{2}\right)^{\frac{1}{2}}
$$

where $X, Y$ and $Z$ represent the magnetic north component, the magnetic east component and the vertical component, respectively. Figure $2 \mathrm{~b}$ and $2 \mathrm{~d}$ shows the baseline-subtracted $\mathrm{X}, \mathrm{Y}$ and $\mathrm{Z}$ component (the daily variations of magnetic field). The horizontal dotted lines in Figure $2 b$ and $2 d$ indicate the baseline for each magnetic field component. Since we used the SuperMAG database data without subtraction of baseline, the daily variations of magnetic field are calculated by subtracting the midnight values (around the local times 22:00 - 02:00) to estimate the dayside magnetic filed variation affected by ionospheric condition caused from the ionization by X-ray or UEV flux. This subtraction manner is well known to be reasonable for the determination of the baseline [24-26].

Figure 2a shows that the response of Total B to the solar flare is negative variation ( -36 $\mathrm{nT})$. Figure $2 \mathrm{~b}$ reveals that the negative variation in $\mathrm{X}$-component results in the negative variation of the Total $\mathrm{B}$. The $\mathrm{X}$-component started decreasing before sunrise (10:53 UT) due to the counter equatorial electrojet (CEJ). The morning negative $\mathrm{X}$-component variation during the intense solar flare is reported by the past researches [13-16]. The positive enhancement $(+117 \mathrm{nT})$ in the Total B occurred near local noon at Huancayo on September 10 (Figure 2c).
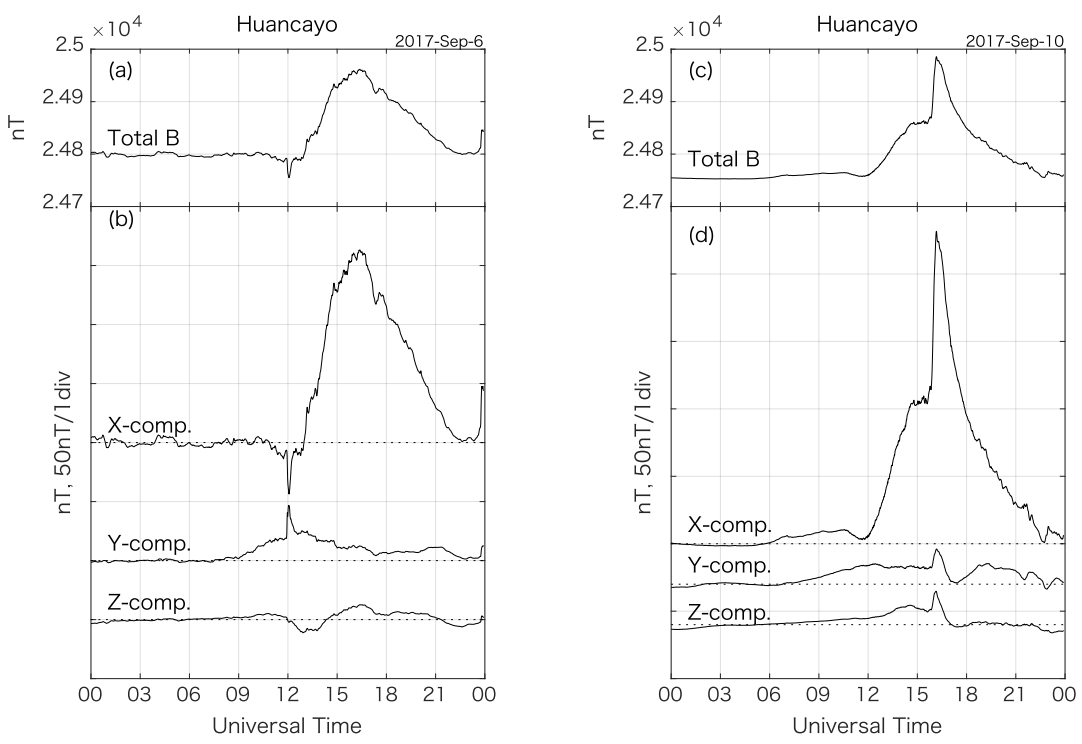

Fig. 2. Magnetometer time series data observed at Huancayo. The left panel (a,b) for September 6, the right panel (c, d) for September 10. (a, c) show the Total B of magnetic field variation, $(b, d)$ show the baseline-subtracted $\mathrm{X}, \mathrm{Y}$ and $\mathrm{Z}$ component. The horizontal dotted line is each baseline calculated from the midnight values.

\section{Analysis and Results}

We demonstrated the magnetic field variation (gsfe) due to the ionospheric disturbance resulting from solar flares, the delay time $\left(\Delta t_{s t n}\right)$ from the onset of X-ray solar flare and the duration $\left(\Delta T_{s t n}\right)$ of gsfe, in Figure 3. The induced amplitude of Total B $\left(\Delta B_{s t n}\right)$ time series data for each magnetometer station during solar flares is defined as

$$
\Delta B_{s t n}=B_{p e a k, s t n}-B_{p r e, s t n}
$$


where $B_{\text {peak,stn }}$ is the peak amplitude of $B_{\text {stn }}$ during the solar flare period, $B_{\text {pre }, \text { stn }}$ is the previous amplitude of $B_{s t n}$, where the value of $B_{s t n}$ rapidly increase or decrease. The delay time $\left(\Delta t_{s t n}\right)$ is calculated as

$$
\Delta t_{s t n}=T_{p r e, s t n}-T_{s}
$$

where $T_{S}$ is the start time of the X-ray flux increase, as shown in Figure 1 and listed in Table 1. $T_{\text {pre }, s t n}$ is the time of $B_{\text {pre }, s t n} . \Delta T_{\text {stn }}$ is the difference between $T_{\text {prestn }}$ and $T_{\text {peak,stn }}$ when the $B_{\text {stn }}$ rises to the peak.
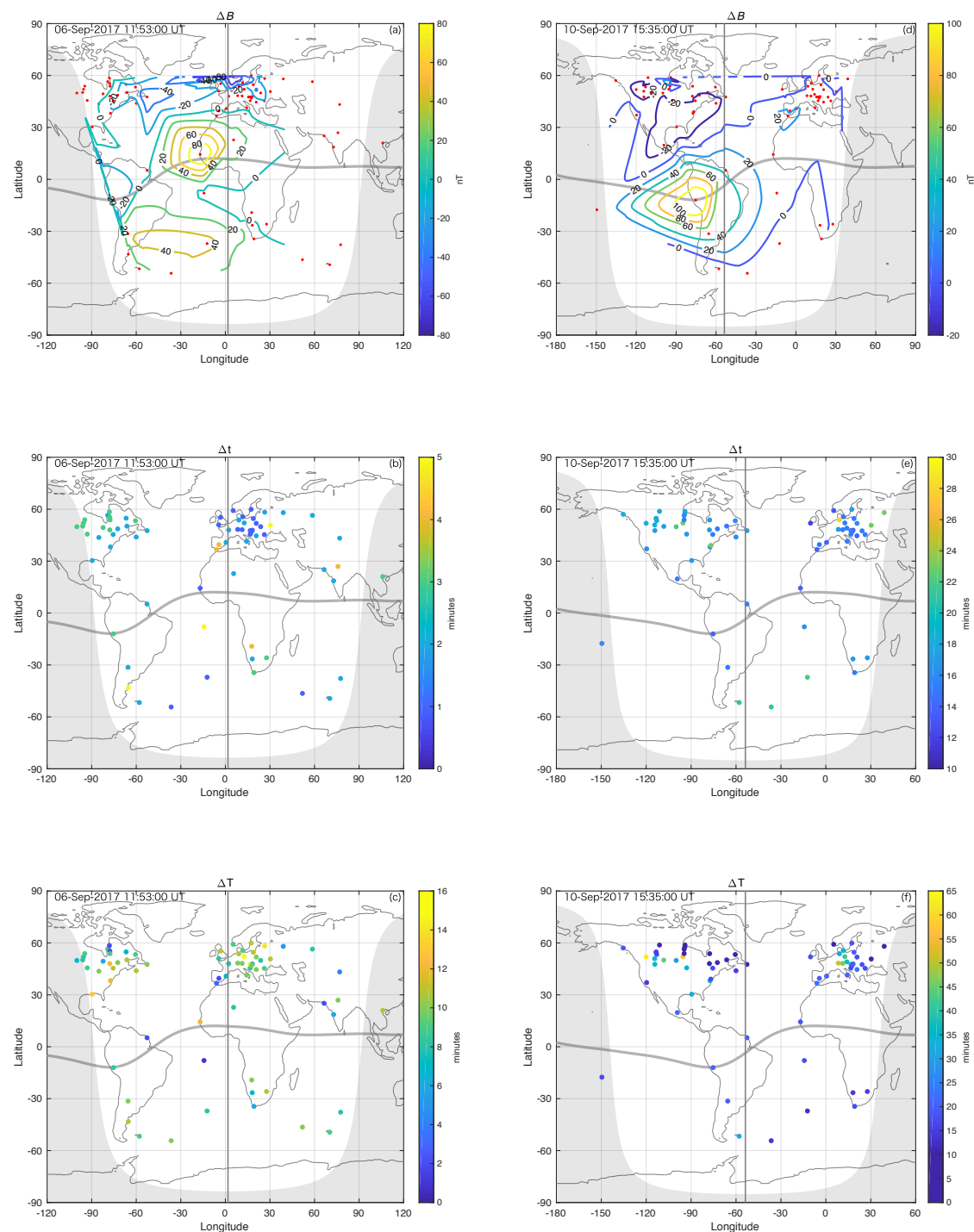

Fig. 3. Ground-based magnetic field response (gsfe) to the intense solar flare. The left panels (a, b, c) for September 6, the right panels (d, e, f) for September 10. (a, d) the amplitude of magnetic field due to the effect of solar flare, $(b, e)$ the time delay [minutes] of gsfe from the start time of X-ray flux increase, and (c, f) the duration [minutes] between the start time and the peak time of gsfe. The red 
dots in $(\mathrm{a}, \mathrm{d})$ indicate the used magnetometer stations in this work. The grey curved horizontal line shows the magnetic equator. The grey shading shows night side at the start time of X-ray flux, which is indicated at the upper left corner of the panel. The grey vertical lines show local noon.

Figure $3 \mathrm{a}$ and $3 \mathrm{~d}$ reveal that the dayside $\Delta B$ increase around the noon sector along the magnetic equator. The negative amplitudes are observed around the dayside highest latitude in the north hemisphere. The equatorial morning region in Figure 3a only shows the negative variation. All $\Delta B$ enhanced the magnetic field variation due to the normal Sq current. of As shown in Figure $3 \mathrm{~b}$ and $3 \mathrm{e}$, the delay time $\Delta t$ is short near noon region and long around morning and evening regions. On September 6, gsfe occurred within 5 minutes from the start time of the X-ray flux increase. The $\Delta t$, for September 10, covers a spread of 16 minutes $(\Delta t$ : $12-28$ minutes). Figure $3 \mathrm{c}$ and $3 \mathrm{f}$ show that the duration $\Delta T$ of gsfe at high latitude is longer than the lower latitude except for the morning higher region. As shown in Figure 4, most of $\Delta t$ agrees the onset time of EUV flux enhancement, but few stations recorded gsfe before EUV flux increase. From histogram $\Delta T$, the duration of gsfe is longer than the duration from start time to peak time of EUV flux and X-ray flux. Finally, there is no response in night side magnetometer data.
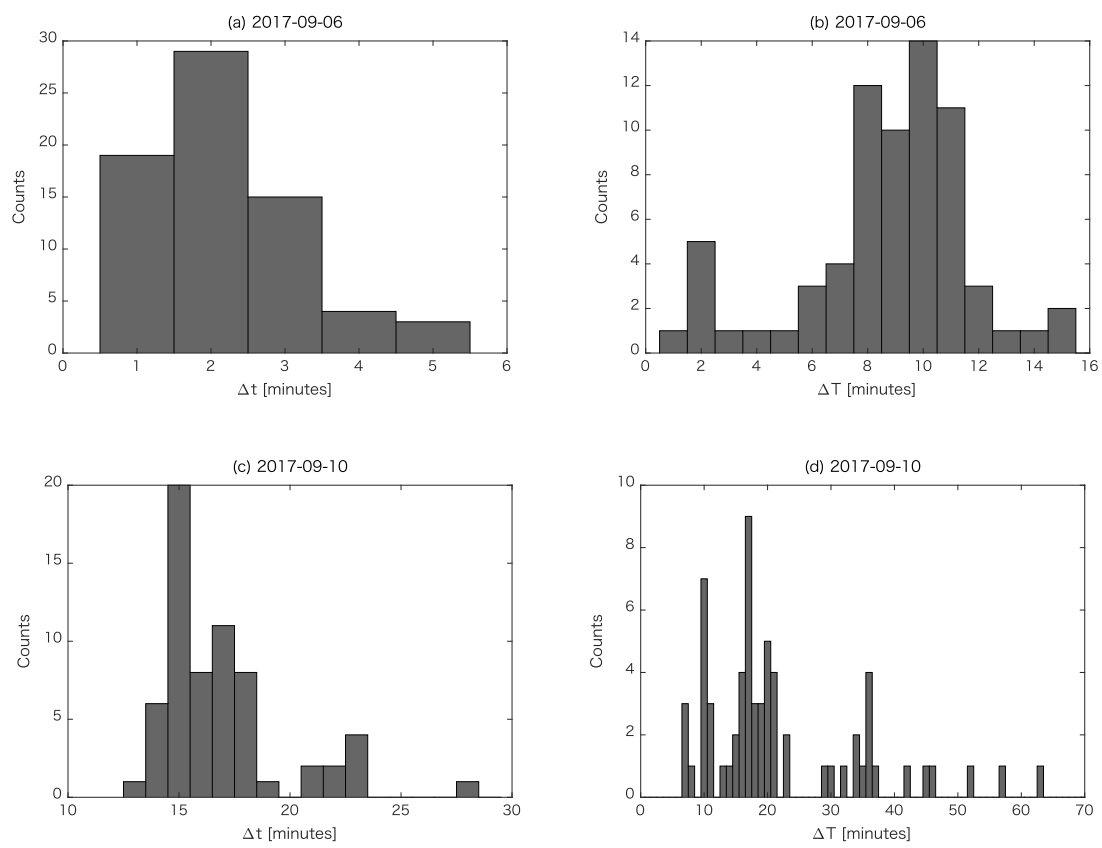

Fig. 4. Histgram of $\Delta t$ and $\Delta T$. The upper panels $(\mathrm{a}, \mathrm{b})$ for September 6 , the bottom panels $(\mathrm{c}, \mathrm{d})$ for September 10 .

\section{Discussion and Summary}

In this paper, we examined the response of global total magnetic field variation $(\Delta B)$, the delay time $(\Delta t)$ from the start time of X-ray flux increase, and the duration of gsfe. As shown in Figure 3, these three parameter doesn't seem to be correlated with the solar zenith angle. Figure 5 shows the dependence of $\Delta B$ to the solar zenith angle. It is clear that the the 
distribution of gsfe differs from that of $\triangle T E C$ against the solar zenith angle (SZA). The $\triangle T E C$ responses linearly correlate to the solar zenith angle [10]. The magnetic equatorial gsfe is largest than other latitude stations. Except for the equatorial gsfe, sgfe has two peaks at around $\mathrm{SZA}=40-60$ degree and $\mathrm{SZA}=60-80$. The sgfe at $\mathrm{SZA}=40-60$ (first peak) is related to the near local noon, the morning gsfe gives the the second peak. The evening gsfe is lower amplitude. This suggests that the gsfe has the significant dependence on local time rather than on the solar zenith angle. Consequently, the main source of gsfe is not only the ionization of ionosphere due to the EUV flux enhancement but also the modulation of ionospheric current system due to the sudden ionospheric disturbances. It might be inferred from the distributions of $\Delta t$ and $\Delta T$, as shown in Figure 3, that the gsfe response is not simply affected by the EUV flux enhancement. Meza et al. (2009)[27] describes that since the ionosphere altitude related to TEC last ionizing longer than the region of gsfe, the generation sources of gsfe and isfe ( $\triangle T E C$ enhancement) remain the different ionospheric altitude (D/Elayer or F-layer).

During X2.8 solar flare on 13 May 2013, the 70-minute blackout of HF wave in ionogram was recorded [28]. In this work, during two intense X-class solar flare, the blackout of HF wave were found in the ionogram at sunlit hemisphere. Figure 6 shows the example of Jixamarca foF2 [MHz] on 6 and 10 September 2017. The ionograms at night side stations have no response on X-ray flux. The duration of blackout depends on the location (local time and the latitude).
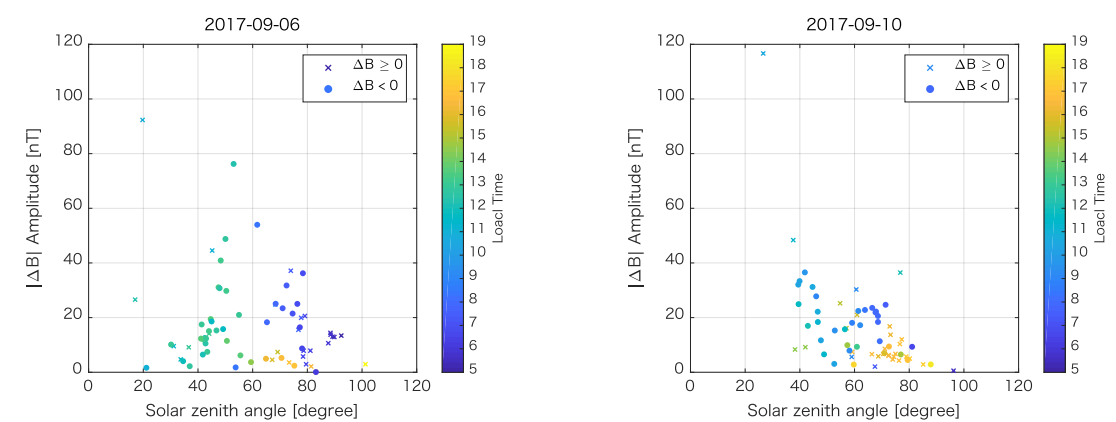

Fig. 5. The relationship between the amplitude of $|\Delta B|$ and the solar zenith angle. Dots show the negative variation of $|\Delta B|$ and cross marks show the positive variation of $|\Delta B|$. The marker color indicates the local time [hour].
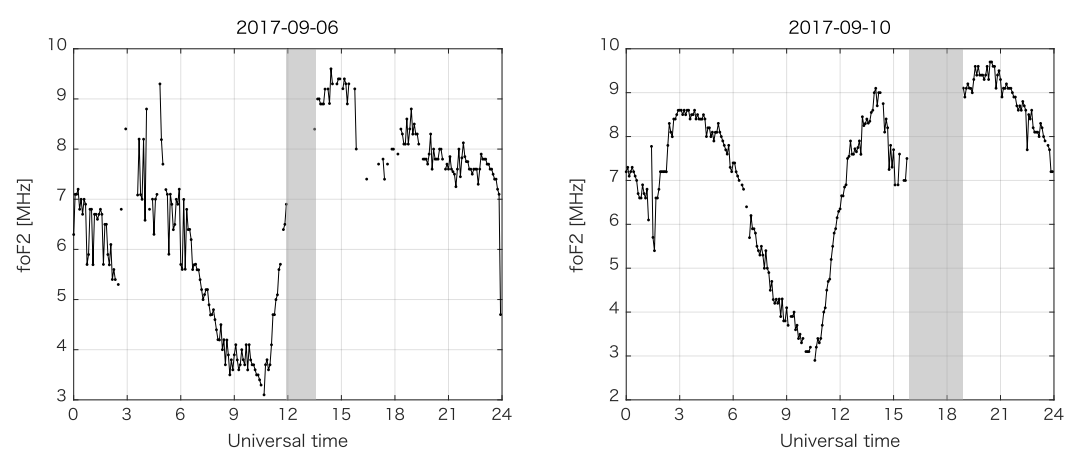

Fig. 6. Time series of foF $2[\mathrm{MHz}]$ at Jicamarca (Peru). The shading shows the blackout of echoes in ionogram.

As shown in Figure 2, each component of gsfe has different variation. A further direction of this work will be to provide the detail examination of $\mathrm{Sq}$ current system using all 
component of magnetic field variation, comparing them to the ionospheric parameter (foF2 and $\triangle T E C$ ). Over the past 17 years, $127 \mathrm{X}$-class solar flares occurred. Further research on these X-class solar flares would clarify the main source and generation mechanism of gsfe, especially the magnetic equatorial negative SFE.

\section{Acknowledgement}

The authors are very grateful to MAGDAS for Huancayo station and SuperMAG (http://supermag.jhuapl.edu/mag/) for other geomagnetic data (McMAC Chain, EMMA, MACCS, IMAGE Chain). We acknowledge NOAA for providing GOES X-ray flux data (https://www.ngdc.noaa.gov/stp/satellite/goes/dataaccess.html) and SOHO/SEM team for providing EUV flux data (https://dornsifecms.usc.edu/space-sciences-center/download-sem-data). We acknowledge the IGP/JRO for providing the Jicamarca Ionosonde data. This work is supported by the JSPS KAKENHI (17J40136).

\section{References}

1. O. K. Garriot, A. V. da Rosa, M. J. Davies, O. G. Villard Jr., J. Geophys. Res., 72, 6099_ 6103, doi:10.1029/JZ072i023p06099 (1967)

2. G. D. Thome, L. S. Wagner J. Geophys.Res., 76, 6883-6895, doi:10.1029/ JA076i028 p06883 (1971)

3. M. Mendillo J. A. Klobuchar R. B. Fritz A. V. da Rosa L. Kersley K. C. Yeh B. J. Flaherty S. Rangaswamy P. E. Schmid J. V. Evans J. P. Schödel D. A. Matsoukas J. R. Koster A. R. Webster P. Chin, J. Geophys. Res., 79(4), 665-672, doi: 10.1029/JA079i004p00665 (1974)

4. R. G. Rastogi, B. M. Pathan, D. R. K. Rao, T. S. Sastry, J. H. Sastri, Earth Planets Space, 51, 947-957 (1999)

5. N. R. Thomson, C. J. Rodger, R. L. Dowden, Geophys. Res. Lett., 31, L06803, doi:10.1029/2003GL019345 (2004)

6. J. Y. Lui, C. S. Chiu, C. H. Lin, J. Geophys. Res., 101(A5), 10855-10862 (1996)

7. T. Nagata, J. Geophys. Res., 57, 1-14 (1952)

8. K. K. Mahajan, N. K. Lodhi, A. K. Upadhayaya, J. Geophys. Res., 115, A12330, doi:10.1029/2010JA015576 (2010)

9. H. Le, L. Liu, H. He, W. Wan, J. Geophys. Res., 116, A11301, doi:10.1029/2011 JA016704 (2011)

10. H. Le, L. Liu, Y. Chen, W. Wan, J. Geophys. Res. Space Physics, 118, 576-582, doi:10.1029/2012JA017934 (2013)

11. W. H. Campbell, Introduction to Geomagnetic Fields, 2nd ed., 102, (Cambridge Univ. Press, Cambridge, U. K. 2003)

12. R. G. Rastogi, V. L. Patel, Proc. Indian Acad. Sci., 82, 121- 141 (1975)

13. R. G. Rastogi, M. R. Deshpande, N. S. Sastri, Nature, 258, 218- 219 (1975)

14. J. H. Sastri, Ann. Geophys., 31, 481- 485 (1975)

15. R. G. Rastogi, J. Atmos. Terr. Phys., 58, 1413- 1420 (1996)

16. R. G. Rastogi, B. M. Pathan, D. R. K. Rao, T. S. Sastry, J. H. Sastri, Earth Planets Space, 51, 947-957 (1999) 
17. Y. Yamazaki, K. Yumoto, A. Yoshikawa, S. Watari, H. Utada, J. Geophys. Res., 114, A05306, doi: 10.1029/2009JA014124 (2009)

18. R.G. Rastogi, H. Chandra, K. Yumoto, Earth Planet Sp 65, 1027, https://doi.org/10.5047/ eps.2013.04.004 (2013)

19. J. W. Gjerloev, J. Geophys. Res., 117, A09213, doi:10.1029/2012JA017683 (2012)

20. K. Yumoto, the 210MM Magnetic Observation Group, J. Geomag. Geoelectr., 48, $1297-$ 1310 (1996)

21. K. Yumoto, the CPMN Group, Earth Planets Space, 53, 981-992 (2001)

22. K. Yumoto, the MAGDAS Group, Solar Influence on the Heliosphere and Earth'sEnvironment: Recent Progress and Prospects, Edited by N. Gopalswamy and A. Bhattacharyya, ISBN-81-87099-40-2, 309-405 (2006)

23. K. Yumoto, the MAGDAS Group, Bull. Astr. Soc. India, 35, 511-522 (2007)

24. A.T. Price, G.A. Wilkins, Philos. Trans. R. Soc. 256, 31-98 (1963)

25. S.R.C. Malin, J.C. Gupta, Geophys. J. R. Astron. Soc. 49, 515-529 (1977)

26. Y. Yamazaki, A. Maute, Space Science Reviews, 206, 299-405, doi:10.1007/s11214016-0282-z. (2017)

27. A. Meza, M. A. Van Zele, M. Rovira, Journal of Atmospheric and Solar-Terrestrial Physics, 71(12), 1322-1332, doi:10.1016/j.jastp.2009.05.015 (2009)

28. P. A. B. Nogueira J. R. Souza M. A. Abdu R. R. Paes J. Sousasantos M. S. Marques G. J. Bailey C. M. Denardini I. S. Batista H. Takahashi R. Y. C. Cueva S. S. Chen, J. Geophys. Res., 120(4), 3021-3032, doi:10.1002/2014ja020823 (2015) 